\title{
High-Speed Mach-Zehnder-OTDR Distributed Optical Fiber Vibration Sensor Using Medium-Coherence Laser
}

\author{
Yuheng TONG, Zhengying LI*, Jiaqi WANG, Honghai WANG, and Haihu YU \\ National Engineering Laboratory for Fiber Optic Sensing Technology, Wuhan University of Technology, \\ Wuhan 430070, China \\ *Corresponding author: Zhengying LI E-mail: zhyli@whut.edu.cn
}

\begin{abstract}
This article presents a high-speed distributed vibration sensing based on Mach-Zehnder-OTDR (optical time-domain reflectometry). Ultra-weak fiber Bragg gratings (UWFBG), whose backward light intensity is $2-4$ orders of magnitude higher than that of Rayleigh scattering, are used as the reflection markers. A medium-coherence laser can substitute conventional narrow bandwidth source to achieve an excellent performance of distributed vibration sensing since our unbalanced interferometer matches the interval of UWFBGs. The $3 \mathrm{~m}$ of spatial resolution of coherent detection and multiple simultaneous vibration sources locating can be realized based on OTDR. The enhanced signal to noise ratio (SNR) enables fast detection of distributed vibration without averaging. The fastest vibration of $25 \mathrm{kHz}$ and the slowest vibration of $10 \mathrm{~Hz}$ can be detected with our system successfully, and the linearity is 0.9896 with a maximum deviation of $3.46 \mathrm{n} \varepsilon$.
\end{abstract}

Keywords: High-speed; Mach-Zehnder-OTDR; ultra-weak fiber Bragg grating array; medium-coherence laser; optical fiber vibration sensing

Citation: Yuheng TONG, Zhengying LI, Jiaqi WANG, Honghai WANG, and Haihu YU, "High-Speed Mach-Zehnder-OTDR Distributed Optical Fiber Vibration Sensor Using Medium-Coherence Laser,” Photonic Sensors, 2018, 8(3): $203-212$.

\section{Introduction}

Distributed optical fiber vibration sensors have received great attention over the past decades for their significant advantages in safety, fully distributed sensing, and large-scale monitoring [1-4]. It is used for a variety of applications including intruder security [5], structure health monitoring [6], mechanical fault diagnostics [7], and oil pipelines leakage monitoring [8], where the signal can be modeled as a spatially resolved temporal disturbance.

Sagnac and Mach-Zehnder interferometers (MZIs) have the advantages of high sensitivity and fast response, but they cannot distinguish multiple simultaneous vibration sources $[9,10]$.

$$
\text { Phase-sensitive optical time-domain }
$$
reflectometry (Ф-OTDR) enables distributed vibration sensing by analyzing the interference of the Rayleigh backscattered light from the vicinity of the coherence length of the pulsed light source when the pulsed light travels along the fiber. However, the scattering light is very weak with typical $-80 \mathrm{~dB}$ scattering ratio, and the required high coherence source [11] causes severe phase noise as well. So its original signal to noise ratio (SNR) is very poor, and temporal-spectral averaging is needed to enhance the SNR [12, 13], which makes this technique only applicable for low speed vibration measurement. For improving the accessible 
frequency response, Mach-Zehnder interferometer and $\Phi-O T D R$ are merged $[14,15] .5 \mathrm{~m}$ of spatial resolution and several $\mathrm{MHz}$ of highest frequency response are realized but the merged systems have no capable of restoring signals of multiple points. Recently, the ultra-weak fiber Bragg grating array is also utilized for high SNR in an improved $\Phi$-OTDR. The reflection is about 4 orders of magnitude higher than that of Rayleigh scattering. But these methods still require a high coherence source: a narrow linewidth distributed feedback fiber laser (DFB-FL) with a linewidth of $5 \mathrm{kHz}$ is employed to ensure the coherence of the reflected light from adjacent FBG [16], and in similar system [17], a tunable laser whose linewidth is $3.7 \mathrm{kHz}$ is selected as the source.

In this paper, we propose a high-speed Mach-Zehnder-OTDR distributed optical fiber vibration sensor based on the ultra-weak fiber Bragg grating (UWFBG). The vibration transducer is a single-mode fiber, and UWFBGs with reflectivity around $0.01 \%$ is written on the fiber to produce reflected lights from different positions. Compared with the previous improved $\Phi$-OTDR based on the UWFBG array, we adjust the optical pulse width to the appropriate value so that the medium-coherence optical pulse interferes with passing through our balanced interference structure. The significant advantage of the scheme proposed here is its low cost of DFB laser of which the linewidth is about $10 \mathrm{MHz}$. In addition, the excellent performances of distributed optical fiber vibration sensing based on medium-coherence light are achieved. Our experimental results show that spatial resolution of vibration locating is $3 \mathrm{~m}$, and the SNR is up to $6.7 \mathrm{~dB}$ without average. Particularly, the detected frequency response is up to $25 \mathrm{kHz}$, and the maximum deviation is about $3.46 \mathrm{n} \varepsilon$.

\section{Principle}

The UWFBG Mach-Zehnder-OTDR distributed vibration sensing system is shown in Fig. 1. The light source is a DFB laser (LUCENT D2526T31) with the maximum output power of $1 \mathrm{~mW}$ and linewidth of $10 \mathrm{MHz}$. The continuous-wave (CW) light from the laser is modulated into nanosecond pulse by semiconductor optical amplifier (SOA, INPHENIX IPSAD1522C) which has an extinction ratio (ER) higher than $70 \mathrm{~dB}$. The pulse light is amplified by an erbium-doped fiber amplifier (EDFA) and then is launched into fiber under test with uniformly distributed UWFBGs by a circulator. The reflected pulses from the UWFBGs go through $3 \times 3$ coupler phase demodulation unit which consists of unbalanced Mach-Zehnder interferometer, $3 \times 3$ coupler, and three detectors. The phase demodulation unit is used to avoid signal fading and restore the amplitude of vibration. The serial data from three detectors are collected by a high-speed oscilloscope.

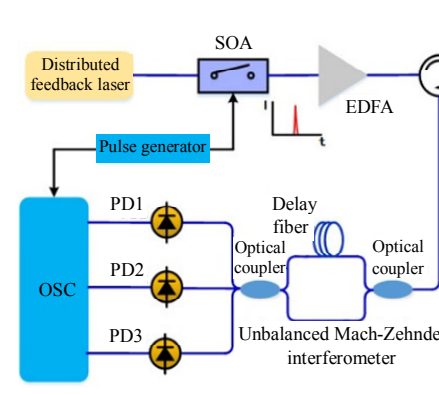

Fig. 1 Schematic of high-speed Mach-Zehnder-OTDR distributed optical fiber vibration sensor using medium-coherence laser. SOA: semiconductor optical amplifier, EDFA: erbium-doped fiber amplifier, CIR: circulator, PD: photodetector, and OSC: oscilloscope.

\subsection{Theory of vibration source locating}

The basic theory locating the position of vibration source is based on the OTDR technology. Like any OTDR system, a short light pulse is fed into the UWFBG array. There is a fixed distance of $L_{1}$ between the adjacent gratings, and delay $\Delta T_{1}$ of adjacent pulses can be written as

$$
\Delta T_{1}=n \cdot \Delta L / c=2 n L_{1} / c
$$

where $n$ is the refractive index of the fiber, $c$ is the velocity of light in vacuum, and $\Delta L$ is the optical path difference (OPD) of pulses reflected by adjacent gratings. The width $\tau$ of optical pulse should be less than the delay $\Delta T_{1}$ to avoid multiple reflected pulses aliasing. Unbalanced path of the MZI in phase demodulation unit separates each 
reflected pulse to two pulses. Making the OPD of the MZI equal to $2 L_{1}$, transmission delay $\Delta T_{2}$ of two arms of MZI can be calculated as

$$
\Delta T_{2}=n\left(L_{2}-L_{3}\right) / c=2 n L_{1} / c=\Delta T_{1}
$$

where $L_{2}$ and $L_{3}$ are the lengths of two interference arms, respectively.

As shown in Fig. 2, the slower sub-pulse $A_{2}$ from a closer grating will be coincident with the faster sub-pulse $B_{1}$ from a further grating and then interfere. Phase perturbation between the two adjacent gratings can be demodulated from the interference signals. Consequently, the correspondence between interference signals and sensing position can be established, and the position of multiple sensing points can be located simultaneously, as shown in Fig. 3.

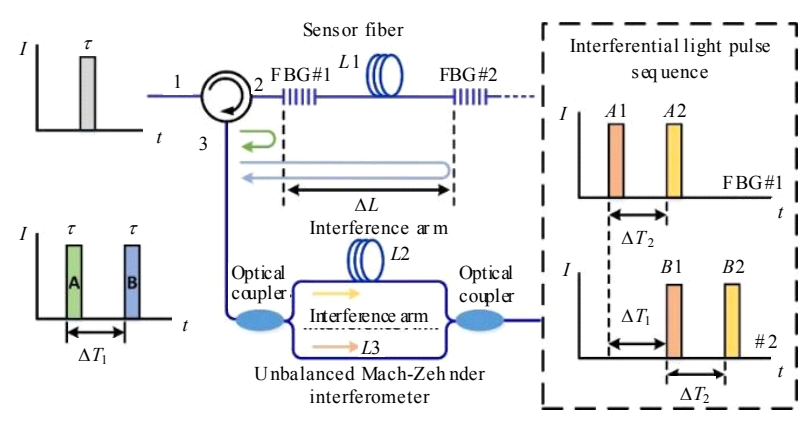

Fig. 2 Principle of vibration source positioning. $\Delta T_{1}$ is the delay of reflection from FBG\#1 and FBG\#2, and $\Delta T_{2}$ is the delay of optical pulse traveling through the unbalanced MZI. $\tau$ is the width of optical pulse.

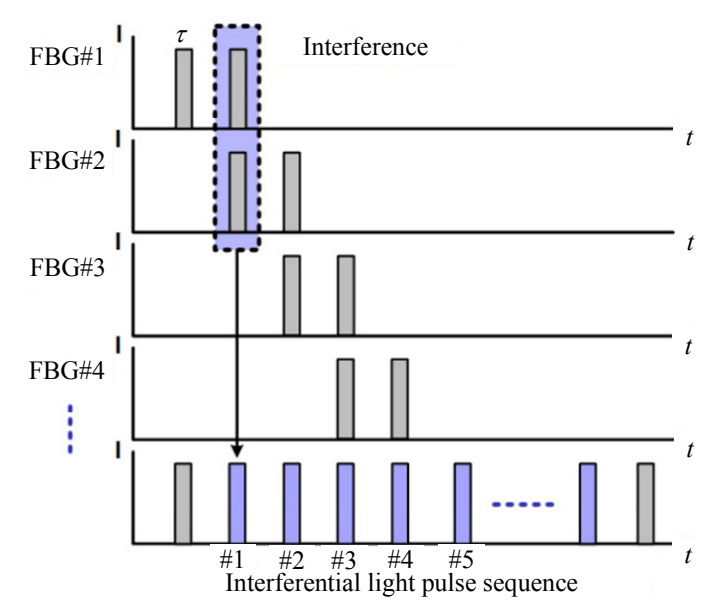

Fig. 3 Relationship between the interferential light pulse sequence and sensing position. The first and last optical pulses of sequence are useless to record vibration. $\# 1$ to $\# 5$ are the serial numbers of sensing fiber segments, respectively.

\subsection{Theory of vibration sensing and demodulation}

In contrast to Rayleigh backscattered light of each fiber section in the conventional $\Phi$-OTDR system, which is attributed by the addition of random distributed scatters within the section length, the reflection of the grating can be regarded as a fixed point reflector. Theoretically, the OPD of each pair of interferential light pulses can be made zero. Thus, the system we propose does not require a high coherence laser.

The intensity of interferential light pulse signal is written as

$$
I=I_{1}+I_{2}+2 \sqrt{I_{1} I_{2}} \cos \left(\Delta \phi+\varphi_{0}\right)
$$

where $I_{1}$ and $I_{2}$ are the intensities of the two optical pulses arriving at the observation point, respectively, $\Delta \phi$ is the dynamic phase shift, and $\varphi_{0}$ is the initial phase. The sensitivity of $\Delta \phi$ fades to zero when $\varphi_{0}$ is close to multiples of $\pi$ [18]. A $3 \times 3$ coupler is chosen to solve this problem. At ideal split ratio, the output intensity of the three arms of $3 \times 3$ coupler can be expressed as follows:

$$
I_{n}=I_{1}+I_{2}+2 \sqrt{I_{1} I_{2}} \cos \left[\begin{array}{c}
\left.\Delta \phi(t)-(i-1) \frac{2}{3} \pi\right], \\
i=1,2,3 .
\end{array}\right.
$$

There is a phase difference of $2 \pi / 3$ between each output port of the $3 \times 3$ coupler. Under no circumstances can the variation ratios of each port output be 0 simultaneously, so we can detect the three output signals to demodulate the $\Delta \phi$ by using the algorithm [19].

When external disturbance is applied on the fiber, the OPD between the two UWFBGs based on the demodulated dynamic phase shifts, and the vibration signal can be restored.

\subsection{Analysis of frequency response}

In a distributed vibration sensing (DVS) system, the significant factor which limits the frequency response of the vibration measurement is the highest sampling frequency.

External vibration and other environmental 
factors can both cause the phase shift $\Delta \phi$. Nevertheless, compared with the external vibration, the environmental factors, such as temperature, have a lower frequency. Thus, $\Delta \phi$ can be expressed as

$$
\Delta \phi(t)=D \cos \omega_{s} t+\varphi(t)
$$

where $D$ and $\omega_{s}$ are the amplitude and angular frequency of phase shift caused by external vibration, and $\varphi(t)$ is the lower frequency phase shift caused by other environmental factors.

The index $i$ is set to 1 . Then substituting (5) into (4), the alternating component (AC) of (4) is rewritten as follows:

$$
I_{\mathrm{AC}}=2 \sqrt{I_{1} I_{2}} \cos \left[D \cos \omega_{s} t+\varphi(t)\right] .
$$

According to Bessel function, we can acquire (7) from (6). In order to restore the vibration signal, enough harmonic information, of which the Bessel value is greater than 0.1 , should be obtained. So, as for different $D$, the relationship between $I_{\mathrm{AC}}$ and $\omega_{s}$ is appeared clearly in Fig. 4(a).

$$
\begin{aligned}
I_{\mathrm{AC}}= & 2 \sqrt{I_{1} I_{2}} \times \\
& \left\{\left[J_{0}(D)+2 \sum_{k=1}^{\infty} J_{2 k}(D) \cos 2 k \omega_{s} t\right] \cos \varphi(t)-\right. \\
& {\left.\left[2 \sum_{k=1}^{\infty}(-1)^{k-1} J_{2 k-1}(D) \cos (2 k-1) \omega_{s} t\right] \sin \varphi(t)\right\} . }
\end{aligned}
$$

The value of $D$ is determined by the sensitivity characteristics of sensor. Here, we do not discuss the sensitivity enhancement or weakening, so $D$ can be considered as 1. From Fig. 4(a), it can be got that the occupied bandwidth of optical intensity variation is twice of the angular frequency of the vibration. In accordance with the Nyquist sampling theorem, the sampling frequency must be more than twice of the occupied bandwidth of the interference optical signal to avoid spectrum aliasing. So if the external vibration has a frequency of $f_{s}$, the lowest sampling frequency required is $4 f_{s}$.

In our DVS system, the repetition rate $f_{\tau}$ of pulse light determines the sampling frequency $f_{s}$. When $f_{\tau}$ is fast enough, there are more than one pulse lights traveling along the sensor fiber with UWFBG at the same moment. Hereby, the multiple-pulse crosstalk will occur. According to Fig. 3, $N$ UWFBGs will produce $N+2$ pulses after MZI in our system. In consideration of avoiding the spectrum aliasing and crosstalk, $f_{\text {DVS }}$ should satisfy the following equation:

$$
f_{\text {DVS }}=4 f_{\tau} \leq 4 c /\left[2 n(N+1) L_{1}\right] .
$$

With an increase in the sensing fiber distance, $f_{\text {DVs }}$ will decrease rapidly, shown as Fig. $4(\mathrm{~b})$. When the spatial resolution is $3 \mathrm{~m}$ and sensing distance is about $1 \mathrm{~km}$, the sensing bandwidth reaches $25 \mathrm{kHz}$.

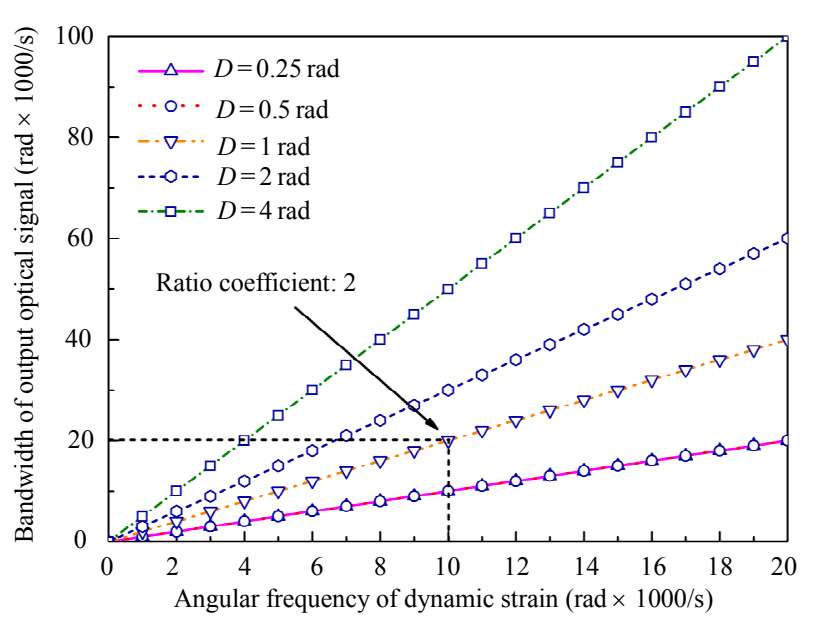

(a)

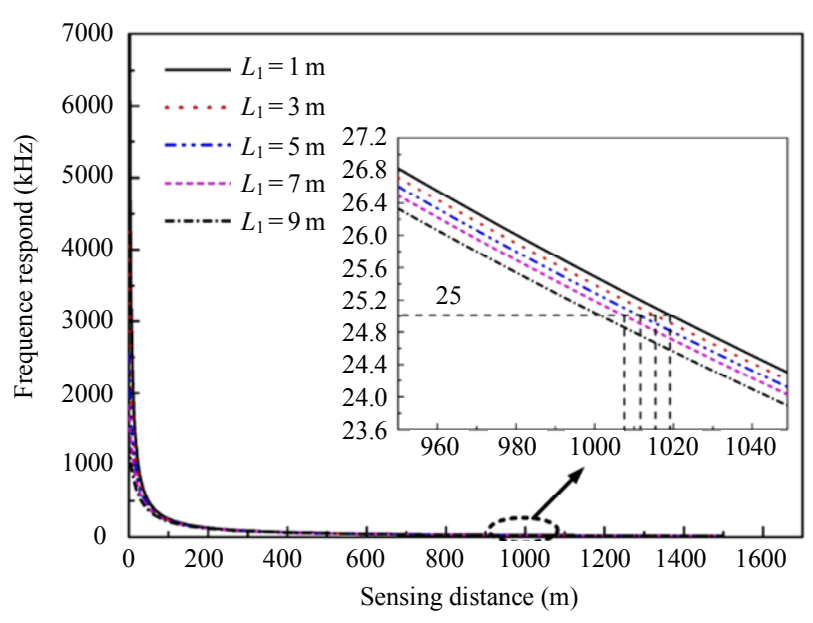

(b)

Fig. 4 Analysis of frequency response: (a) the relationship among the bandwidth of optical signal, the phase shift amplitude of optical signal, and the frequency of the external vibration and (b) the relationship among the frequency response, sensing distance, and the spatial resolution. 


\subsection{Analysis of signal-to-noise ratio}

There are 3 main noises in the system: shot noise $\sigma_{\mathrm{s}}^{2}=2 q \cdot\left(I_{\mathrm{RBS}}+I_{n}+I_{d}\right) \cdot \Delta B$ (here $q$ is the charge of electron, $I_{\mathrm{RBS}}$ is the detected current of the Rayleigh backscattering, $I_{d}$ is the dark current of detector, and $\Delta B$ is the bandwidth of signal), thermal noise $\sigma_{T}^{2}$, and Rayleigh backscattered noise. Assume the scattering coefficient for every scattering center is the same and $M$ scattering centers in each fiber section with length of $L_{1}$. The detected current signal is proportional to the optical intensity, so the Rayleigh backscattered current noise can be regarded as

$$
I_{\mathrm{RBS}} \propto I_{0}(t) \exp \left(-2 \alpha L_{N}\right) \sum_{k=1}^{M} r_{k}^{N} \exp \left[\varphi_{s}(t)+\varphi_{s 0}\right]
$$

where $I_{\mathrm{RBS}}(t)$ is the detected current of the Rayleigh backscattering at distance $L_{N}=N L_{1}, I_{0}(t)$ is the intensity of the incident light, $\alpha$ is the attenuation coefficient of the fiber, and $r_{k}^{N}$ is the Rayleigh reflection coefficient of the scatter center in $N$-th section.

So the SNR of the detected signal is described as

$$
\begin{aligned}
S N R & =\frac{\left\langle I_{\mathrm{AC}}^{2}\right\rangle}{\sigma^{2}} \\
& =\frac{2 R^{2} P_{r}^{2}}{2 R^{2} P_{\mathrm{RBS}}^{2}+2 q\left(P_{\mathrm{RBS}} R+P_{r} R+I_{d}\right) \Delta B+\sigma_{T}^{2}}
\end{aligned}
$$

where $P_{r}$ and $P_{\mathrm{RBS}}$ are the detected powers of reflected light and backscattering light, respectively. $\sigma_{s}^{2}$ and $\sigma_{T}^{2}$ related to the detector have an ultra-low magnitude. Since the reflectivity of the
UWFBG is four orders of magnitude greater than the Rayleigh scattering, $P_{r}$ is much higher than $P_{\mathrm{RBS}}$. For this reason, the high SNR of the system could be achieved.

\section{Experimental results and discussion}

In experimental setup, 332 UWFBGs are distributed over 1-km long fiber with equal interval of $3 \mathrm{~m}$. The UWFBGs have uniform wavelength of $1550.947 \mathrm{~nm}$, as well as bandwidth of $0.2 \mathrm{~nm}$ and reflectivity of $0.01 \%(-40 \mathrm{~dB})$. Fiber sections between UWFBG\#100, UWFBG\#101, UWFBG\#300, and UWFBG\#301 are wound around two identical cylindrical piezoelectric transducers (PZTs) to generate strain variation. The experimental setup includes our proposed DVS system and a calibration component which is a standard Mach-Zehnder interferometer, as shown in Fig. 5. When the DVS system measures, the optical Switch 1 connects DFB laser (Lucent 2526, $10 \mathrm{MHz}$ linewidth) to SOA, and the optical Switch 2 connects UWFBG array to circulator. The wavelength of DFB laser is located at $1550.947 \mathrm{~nm}$ to match the identical UWFBG. The light pulses have a repetition rate of $100 \mathrm{kHz}$, with a pulse width of $20 \mathrm{~ns}$ (obey $\tau<2 n L_{1} / c$ ). The phase demodulation unit adopts an imbalance path of $6 \mathrm{~m}$ for matching the interval of gratings. Data are acquired with an oscilloscope (Keysight DSA 134A) for $50 \mathrm{~ms}$ at 200 Msps. The experimental rig is shown as Fig. 6.

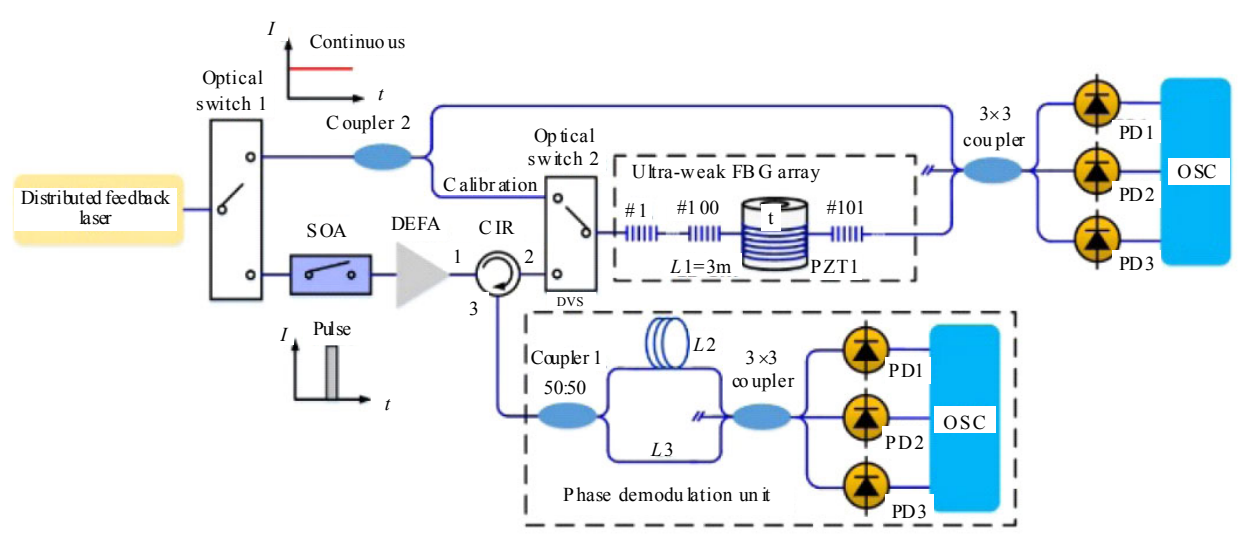

Fig. 5 Structure for experiment, all UWFBGs have same reflectivity and central wavelength. When optical switch 2 connects UWFBG array to coupler 2, a standard Mach-Zehnder interferometer is to calibrate the vibration. 


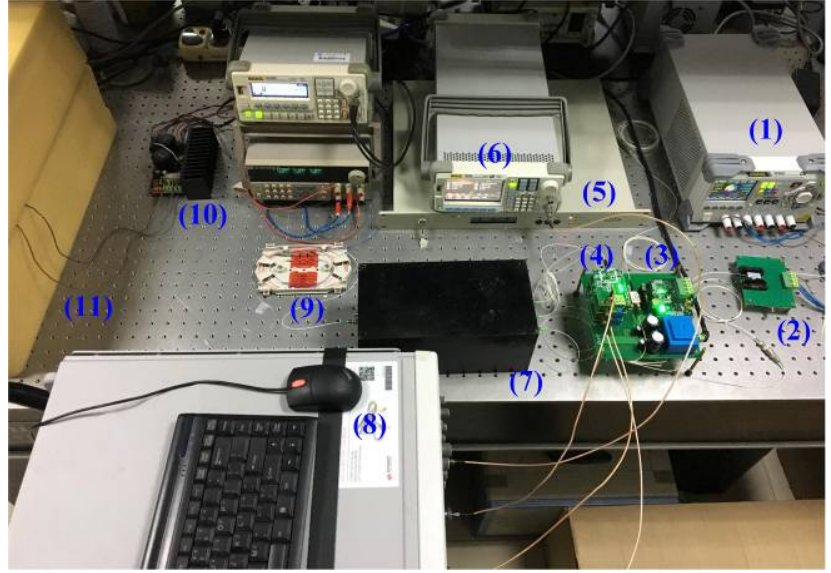

Fig. 6 Experimental rig of our distributed optical fiber vibration sensing system: (1): DC power supply, (2): DFB, (3): SOA, (4): PD, (5): EDFA, (6): pulse generator, (7): unbalanced Mach-Zehnder interferometer, (8): OSC, (9): CIR, (10): PZT driver, and (11): UWFBG array.

To compare the SNR and the coherent requirement of light source, on the one hand, a narrow linewidth laser (NL) and our medium-coherence laser employed in the conventional $\Phi$-OTDR in [13] are compared, and Fig. 7 shows the comparison of linewidth. According to Lorenz fitting curve of self-heterodyne of NL and DFB laser, the full widths at half maximum (FWHM) are $22.3 \mathrm{kHz}$ and $17.8 \mathrm{MHz}$, respectively. Figure 8 shows the results of vibration locating. 20-ns optical pulses and $1.5-\mathrm{km}$ sensing fiber are used for testing. With the averaging times of 10 , the SNR of narrow linewidth laser system is $5.31 \mathrm{~dB}$, but the medium-coherence system cannot locate vibration source due to the awful SNR.

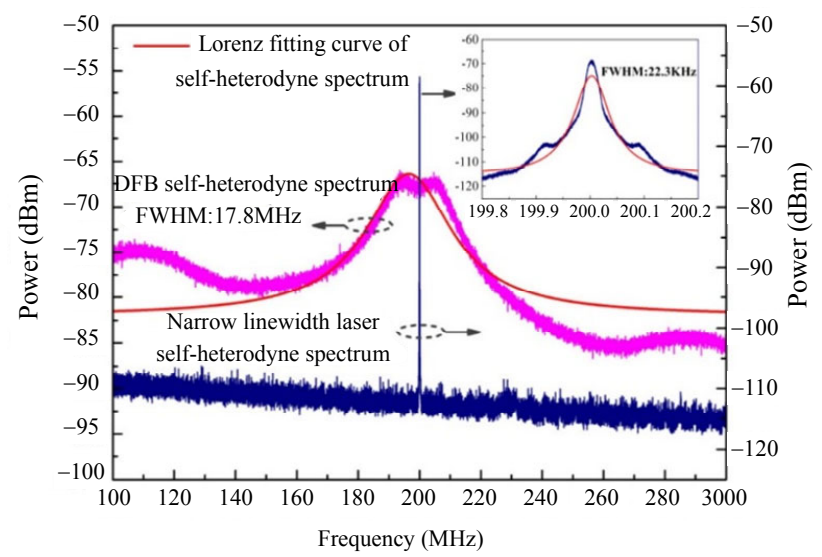

Fig. 7 Linewidth of narrow linewidth and medium-coherence laser.

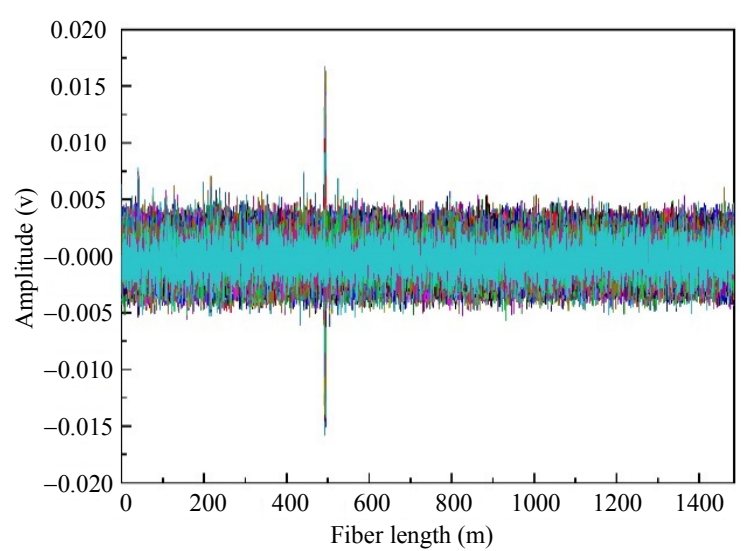

(a)

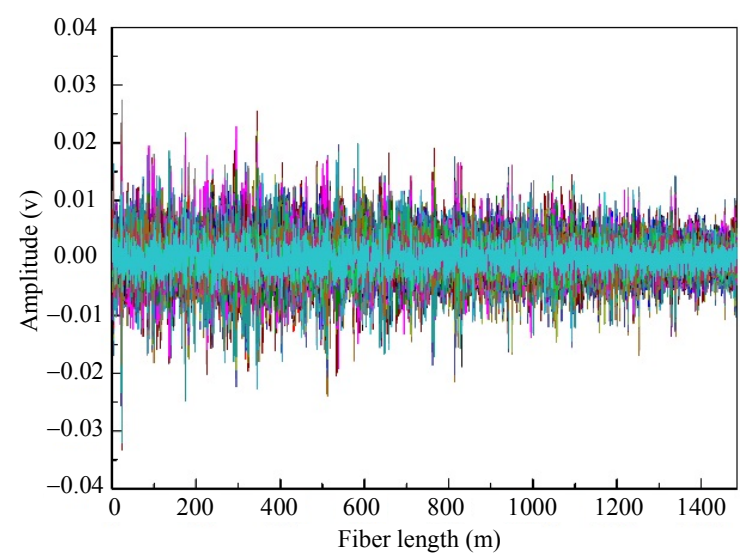

(b)

Fig. 8 Results of $\Phi$-OTDR vibration locating with different light source: (a) the location information for the averaging times of 10 by using a narrow linewidth laser and (b) the location information for the averaging times of 10 by using the same medium-coherence as our system.

On the other hand, when only PZT1 generates vibration, the interferential pulse arrays for 100 pulse periods are detected by PD1. In Fig. 9, 6 interferential pulses which correspond to the distance from $294 \mathrm{~m}$ to $312 \mathrm{~m}$ are shown. From the superposition of 100 interferential pulse arrays, it can be seen that the intensity of the interference pulse corresponding to the fiber section where PZT1 is located is greatly fluctuated, while the other pulses do not change much. We extract the peak value of each pulse in all the 5000 detected interferential pulse arrays, and Fig. 10 shows the superposition of intensity fluctuation at each sensing position. According to (10), we can calculate the SNR by analyzing the intensity fluctuation amplitude $A_{\text {signal }}$ in the position with vibration, and 
the $A_{\text {noise }}$ in the position without vibration, which is $10 \lg \left(A_{\text {signal }} / A_{\text {noise }}\right) . A_{\text {signal }}=1.12 \mathrm{~V}$ and $A_{\text {noise }}=0.24 \mathrm{~V}$, so SNR is $6.7 \mathrm{~dB}$. Therefore, our system can locate the vibration clearly without multiple averages.

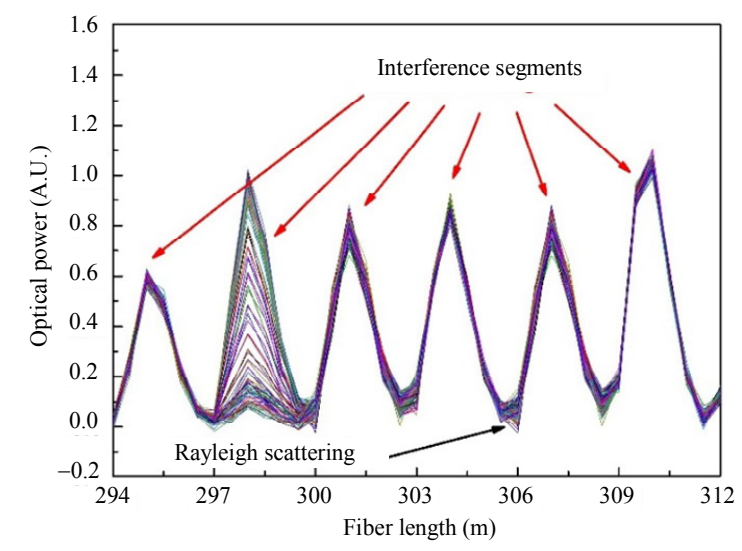

Fig. 9 Parts of superposition of all 100 output optical pulse sequence from PD1 when only PZT1 has a vibration.

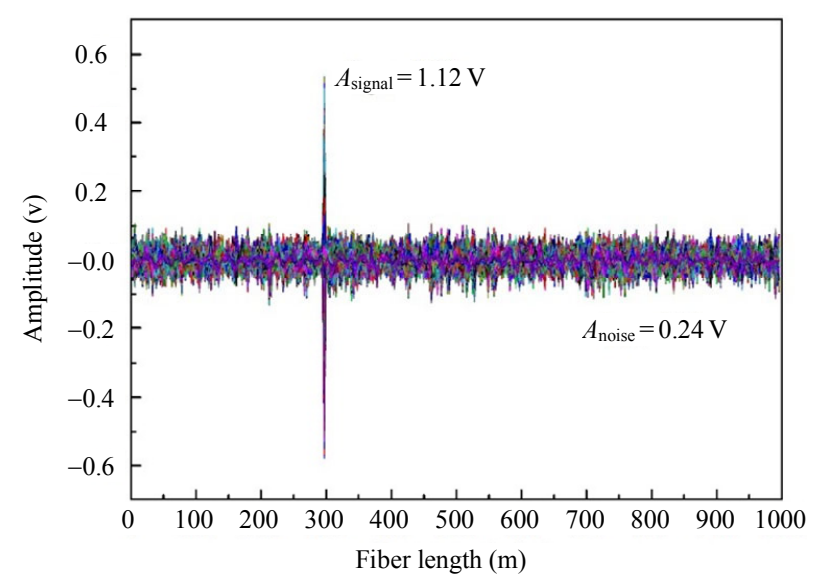

Fig. 10 Location information without average in our proposed system by using the medium-coherence.

Here we use two same PZTs to test the locating and demodulation capability of our DVS system. They generate vibration at different frequencies and amplitudes simultaneously, and the parameters of two sine-wave driven signals are $12 \mathrm{~V}, 1 \mathrm{kHz}$, and $6 \mathrm{~V}, 3 \mathrm{kHz}$, respectively. The dynamic phase shift can be demodulated from the intensity variation of interferential pulse. According to the parameters used in the experiment [19], the measurement sensitivity of OPD $\Delta l$ is $84.2 \mathrm{~nm} / \mathrm{rad}$, and the dynamic strain sensitivity is about $28.06 \mathrm{n} \varepsilon / \mathrm{rad}$. The global demodulation result of $1-\mathrm{km}$ fiber is given in
Figs. 11(a) and 11(b). It can be clearly seen that there exist two magnitude changes located within the darker frame in Fig. 11(a) at distance of $300 \mathrm{~m}$ and $900 \mathrm{~m}$. The two peaks in the Fig. 11(b) correspond to the two vibration regions in the sensing fiber.

Here for wave shape details we just show the time response data from $-2.5 \mathrm{~ms}$ to $2.5 \mathrm{~ms}$ in Fig. 11(c). The amplitudes of dynamic strains detected from PZT1 and PZT2 are $59.7 \mathrm{n} \varepsilon$ and $23.9 \mathrm{n} \varepsilon$, respectively. The results from spectral analysis via fast Fourier transform (FFT) are shown in Fig. 11(d). It can be found that two clear main peaks are at $1 \mathrm{kHz}$ and $3 \mathrm{kHz}$, and the second harmonic suppression ratios of demodulated signal are $43.9 \mathrm{~dB}$ and $41.13 \mathrm{~dB}$, respectively.

In order to confirm the deviation of amplitude measurement, linearity and stability experiment have been also performed. At first, we set the frequency of sine-wave driving signal of the vibration source PZT1 to be $1 \mathrm{kHz}$, and the amplitude to be increased from $1.2 \mathrm{~V}$ to $12 \mathrm{~V}$. The vibration is measured with the DVS system and the calibration component respectively, as shown in Fig. 12. Since the values of MZI do not contain a round trip, it is divided by 2 to match the values of DVS. Because the calibration system and our DVS both exist dithering, the results are averaged for 5 times to compare the detection error. Compared with the calibrated values from the calibration component, the maximum measuring error is only $3.46 \mathrm{n} \varepsilon$. It can be found that the measured and calibrated values show a linear relationship, and the linearity of curving fitting is 0.9896 . Secondly, we drive the PZT1 by a stable sine-wave with voltage of $12 \mathrm{~V}$ and frequency of $1 \mathrm{kHz}$, and the dynamic strain every 10 minutes for a total of 100 times is collected by our DVS system. In Fig. 13, the standard deviation of the system is $3.410 \mathrm{n} \varepsilon$. Actually, the amplitude of vibration source is not absolutely stable, so it will also introduce dithering. 


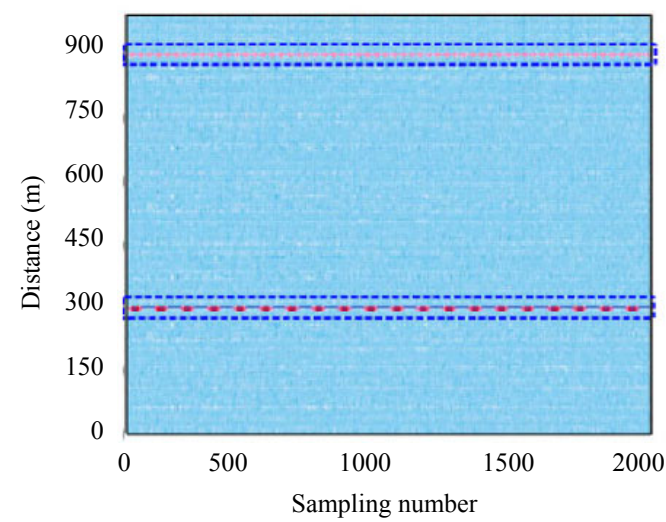

(a)

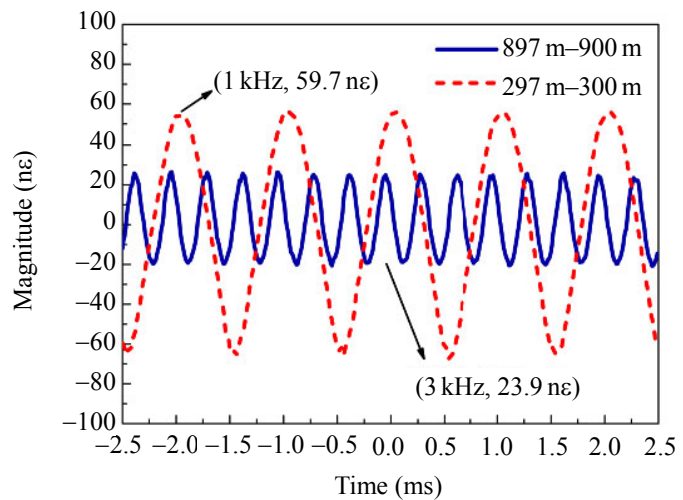

(c)

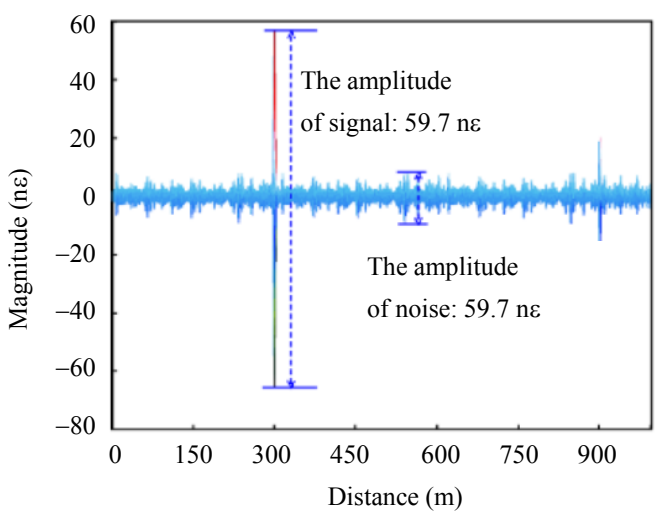

(b)

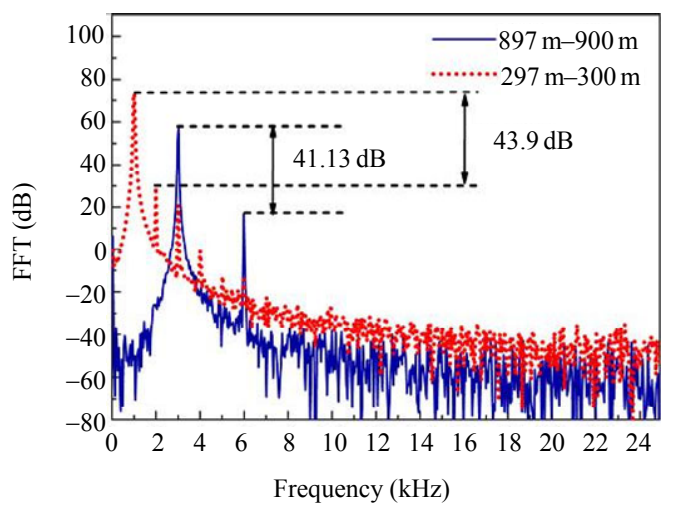

(d)

Fig. 11 Results of DVS experiment: (a) demodulated scalogram of the $12 \mathrm{~V}, 1 \mathrm{kHz}$, and $6 \mathrm{~V}, 3 \mathrm{kHz}$ vibration signal at $300 \mathrm{~m}$ and $900 \mathrm{~m}$, respectively, (b) demodulated phase changing diagram between $300 \mathrm{~m}$ and $900 \mathrm{~m}$. It illustrates the amplitude of dynamic strain at different position, and (c) time responses of our system at $300 \mathrm{~m}$ and $900 \mathrm{~m}$ and (d) frequency responses of the demodulation results at $300 \mathrm{~m}, 900 \mathrm{~m}$, and background.

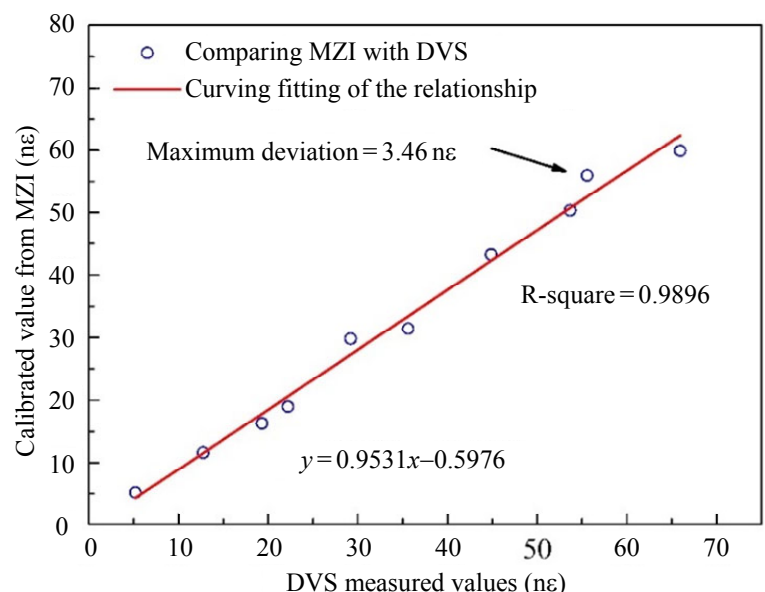

Fig. 12 Comparison between the variations sensing results of DVS and MZI.

Further experiment has been made to test the frequency response of DVS system. In fact, the frequency response of the vibration transducer can also influence the frequency response of system, but the repetition frequency of sampling optical pulse is the most important factor in this experiment. According to the previous theoretical analysis, we set the frequency of PZT1 to be $10 \mathrm{~Hz}$ and $25 \mathrm{kHz}$, respectively. And the clear main peaks at $10 \mathrm{~Hz}$ and $25 \mathrm{kHz}$ respectively are shown in Fig. 14.

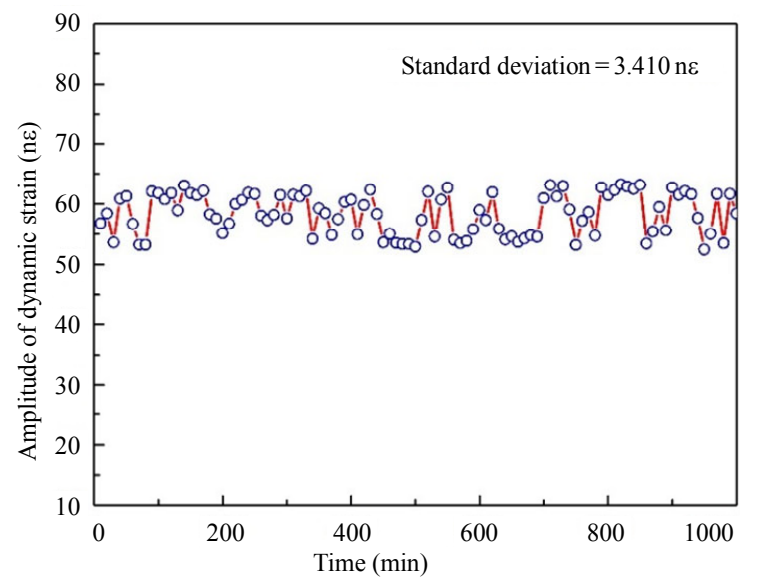

Fig. 13 Demodulated results of 100 dynamic strains in the same environment, and an interval of 10 minutes is set between each results. 


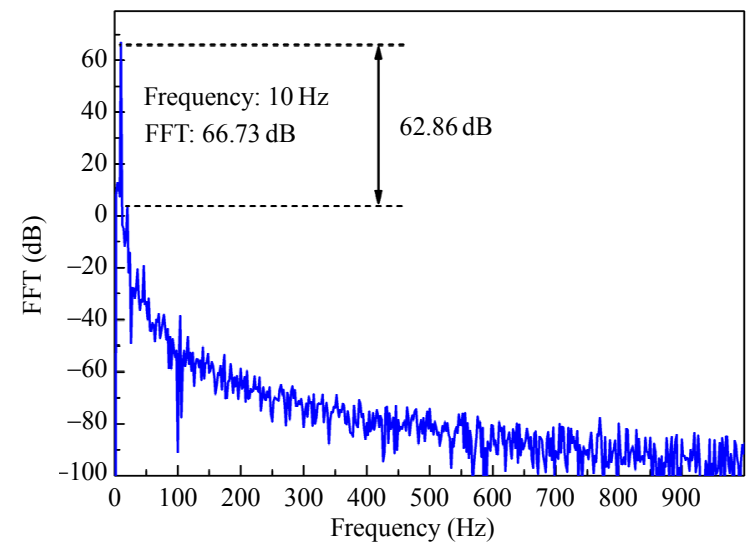

(a)

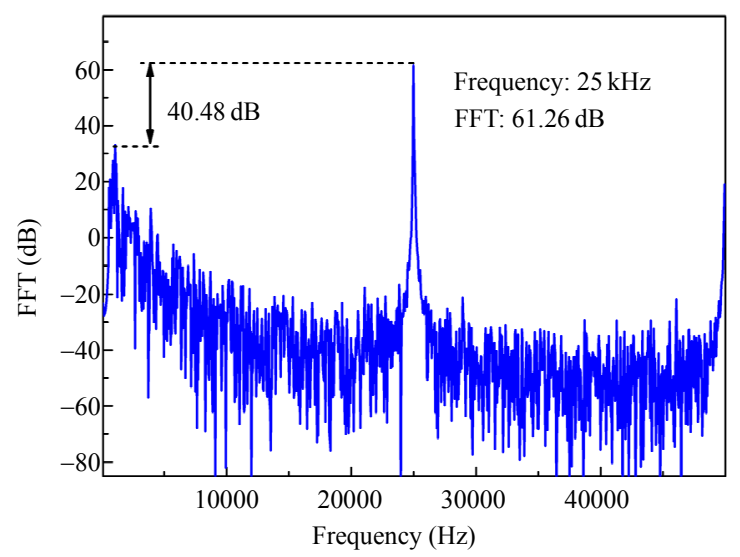

(b)

Fig. 14 Results of frequency response experiments: (a) minimum frequency response of the system for the driving frequency of $10 \mathrm{~Hz}$ and (b) maximum frequency response of the system for the driving frequency of $25 \mathrm{kHz}$.

\section{Conclusions}

In this paper, a Mach-Zehnder-OTDR distributed optical fiber vibration sensing system using medium-coherence laser is proposed. The higher reflection generated by UWFBG array is adopted instead of the Rayleigh backscattering (RBS) in the sensing fiber, and the position information can be obtained easily by OTDR. Based on the balanced Mach-Zehnder structure, the medium-coherence laser DFB can be used as light source to realize an excellent SNR. In our experiments, the vibration sensing signal with a high SNR of $6.7 \mathrm{~dB}$ can be obtained without averaging. The demonstrated setup has a sampling frequency of $100 \mathrm{kHz}$ for vibration and improves the highest frequency response to
$25 \mathrm{kHz}$ in this monitor system. Spatial resolution of $3 \mathrm{~m}$ and simultaneous localization of multiple vibration sources are achieved in a $1-\mathrm{km}$ long sensing fiber by detecting the delay of the pulse in time domain. A $3 \times 3$ coupler is used to restore the amplitude of the vibration signal, and crosstalk between neighboring sensing position is weak enough. The maximum measuring error is only $3.46 \mathrm{n} \varepsilon$ compared with the standard Mach-Zehnder interferometer, and the linearity of measurement is 0.9896 .

\section{Acknowledgment}

This work was supported in part by the National Natural Science Foundation of China (Grant No. 61735031), Natural Science Foundation of Hubei Province of China (Grant No. 2018CFA056), and the Excellent Dissertation Cultivation Funds of Wuhan University of Technology (Grant No. 2017-YS-057).

Open Access This article is distributed under the terms of the Creative Commons Attribution 4.0 International License (http://creativecommons.org/licenses/by/4.0/), which permits unrestricted use, distribution, and reproduction in any medium, provided you give appropriate credit to the original author(s) and the source, provide a link to the Creative Commons license, and indicate if changes were made.

\section{References}

[1] X. Bao, D. P. Zhou, C. Baker, and L. Chen, "Recent development in the distributed fiber optic acoustic and ultrasonic detection," Journal of Lightwave Technology, 2017, 35(16): 3256-3267.

[2] Z. Y. Li, M. Y. Liu, Y. M. Wang, Q. Liu, and J. M. Gong, "Delay calibration method for wavelength-swept laser-based FBG demodulation system," IEEE Photonics Technology Letters, 2014, 26(20): 2090-2092.

[3] Z. H. Luo, H. Q. Wen, H. Y. Guo, and M. H. Yang, "A time- and wavelength-division multiplexing sensor network with ultra-weak fiber Bragg gratings," Optics Express, 2913, 21(19): 22799-22807.

[4] Z. Y. Li, Z. Q. Xu, Z. H. Tang, M. Zhao, L. J. Cai, 
and Q. Liu, "Research of high-speed FBG demodulation system for distributed dynamic monitoring of mechanical equipment," Advances in Mechanical Engineering, 2013, 7: 679-681.

[5] X. B. Hong, H. X. Guo, and J. Wu, "A Brillouin optical time domain analysis based distributed fiber optic intrusion sensor system," Chinese Journal of Lasers, 2010, 37(37): 1037-1041.

[6] Y. L. Lu, T. Zhu, L. Chen, and X. Y. Bao, "Distributed vibration sensor based on coherent detection of phase-OTDR," Journal of Lightwave Technology, 2010, 28(22): 3243-3249.

[7] A. Gunday and S. E. Karlik, "Optical fiber distributed sensing of temperature, thermal strain and thermo-mechanical force formations on OPGW cables under wind effects," in Proceeding of International Conference on Electrical and Electronics Engineering, Bursa, Turkey, 2013, pp. 14-15.

[8] Y. N. Wang and Z. D. Jiang, "Application of Golay codes to distributed optical fiber sensor for long-distance oil pipeline leakage and external damage detection," Chinese Optics Letters, 2006, 4(3): 141-144.

[9] C. Y. Ma, T. G. Liu, K. Liu, J. F. Jing, Z. Y. Ding, L. Pan, et al., "Long-range distributed fiber vibration sensor using an asymmetric dual Mach-Zehnder interferometers," Journal of Lightwave Technology, 2016, 34(9): 2235-2239.

[10] C. X. Zhang, Q. Li, S. Liang, W. T. Lin, L. J. Li, and X. Zhang, "Location algorithm for multi-disturbances in fiber-optic distributed disturbance sensor using a Mach-Zehnder interferometer," in Proceeding of the 9th International Conference on Optical Communications and Networks, Nanjing, China, 2010, pp. 103-107.

[11] J. C. Juarez and H. F. Taylor, "Distributed fiber optic intrusion sensor system," Journal of Lightwave
Technology, 2015, 23(6): 2081-2087.

[12] Y. Koyamada, M. Imahama, K. Kubota, and K. Hogari, "Fiber-optic distributed strain and temperature sensing with very high measurand resolution over long range using coherent OTDR," Journal of Lightwave Technology, 2009, 27(9): 1142-1146.

[13] A. Masoudi, M. Belal, and T. P. Newson, "A distributed optical fiber dynamic strain sensor based on phase-OTDR," Measurement Science \& Technology, 2013, 24(8): 085204.

[14] T. Zhu, Q. He, X. H. Xiao, and X. Y. Bao, "Modulated pulses based distributed vibration sensing with high frequency response and spatial resolution," Optics Express, 2013, 21(3): 2953.

[15] Q. He, T. Zhu, X. H. Xiao, B. M. Zhang, D. M. Diao, and X. Y. Bao, "All fiber distributed vibration sensing using modulated time-difference pulses," IEEE Photonics Technology Letters, 2013, 25(20): 1955-1957.

[16] C. Wang, Y. Shang, X. H. Liu, C. Wang, H. H. Yu, D. S. Jia, et al., "Distributed OTDR-interferometric sensing network with identical ultra-weak fiber Bragg gratings," Optics Express, 2015, 23(22): 29038-29046.

[17] C. B. Cameron, R. M. Keolian, and S. L. Garrett, “A symmetric analogue demodulator for optical fiber interferometric sensors," in Proceeding of IEEE Symposium on Circuits and Systems, Monterey, CA, USA, 1991, pp. 666-671.

[18] Z. F. Wang, H. Luo, and Y. M. Hu, "Signal detection technique for fiber-optic interferometric sensors," Journal of Applied Optics, 2007, 28(1): 86-91.

[19] F. Zhu, Y. X. Zhang, L. Xia, X. L. Wu, and X. P. Zhang, "Improved $\Phi-O T D R$ sensing system for high-precision dynamic strain measurement based on ultra-weak fiber Bragg grating array," Journal of Lightwave Technology, 2015, 33(23): 4775-4780. 